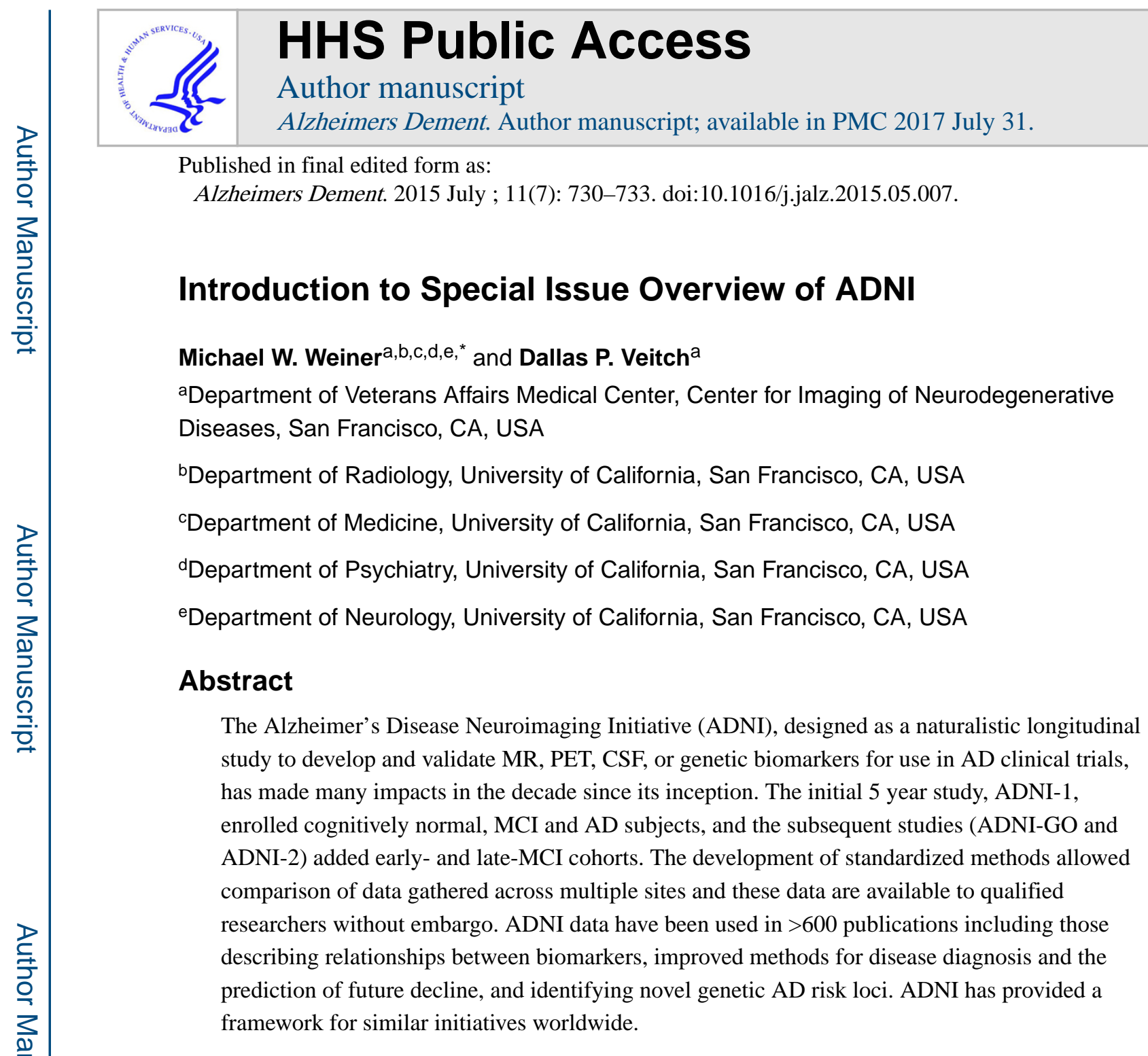

Established in 2004 in response to a need for improved biomarkers in clinical trials of Alzheimer's disease (AD) therapies, the Alzheimer's Disease Neuroimaging Initiative (ADNI) has made, and continues to make a profound impact on nearly all aspects of AD pathobiology and patient oriented research over the past decade The ADNI Team is now looking forward to continuing its work as a longitudinal study incorporating the latest advances in AD research. At the beginning of this century, details of the pathophysiological changes leading to neuronal degeneration in $\mathrm{AD}$ were lacking (1), mild cognitive impairment (MCI) had just been recognized as a prodromal state of the disease (2), and clinical trials of disease modifying treatments were limited by the lack of sensitivity of clinical and cognitive outcome measures to detect subtle treatment effects. The primary goal

\footnotetext{
"Corresponding author. Center for Imaging of Neurodegenerative Diseases, VA Medical Center, San Francisco, 4150, Clement St. (114M), San Francisco, CA 94121. Tel.: 415-221-4810 x3642; Fax: 415-668-2864. michael.weiner@ucsf.edu.

Publisher's Disclaimer: This is a PDF file of an unedited manuscript that has been accepted for publication. As a service to our customers we are providing this early version of the manuscript. The manuscript will undergo copyediting, typesetting, and review of the resulting proof before it is published in its final citable form. Please note that during the production process errors may be discovered which could affect the content, and all legal disclaimers that apply to the journal pertain.

Conflicts of Interest

Dallas P. Veitch has no conflicts of interest to report.
} 
of ADNI therefore was to develop and validate biomarkers that could function as surrogate outcome measures with greater statistical power than clinical or cognitive measures alone in addition to advancing understanding of the pathobiology of $\mathrm{AD}(3,4)$.

\section{History and Governance}

ADNI was initially funded by a combination of public, private and foundation sources as a five-year study of normal cognitive aging, MCI and early AD, enrolling more than 200 elderly control subjects, $400 \mathrm{MCI}$ patients and 200 early AD patients across 56 sites in the US and Canada (3). The initiative was structured as a series of eight cores (Clinical, MRI, PET, Biomarker, Genetics, Neuropathology, Biostatistics, and Informatics) under the direction of an Administrative Core and was overseen by a Steering and Executive Committee consisting of representatives from all funding sources as well as principal investigators of the ADNI sites (5). In addition, a Private Partner Scientific Board convened by the foundation for the National Institutes of Health provided an opportunity for industry partners to exchange study related scientific data in a precompetitive manner, and a Data And Publications Committee monitored scientific publications arising from ADNI research and data (5). A subsequent Grand Opportunities grant funded ADNI-GO which enrolled a new cohort of 200 early MCI patients, and a competitive renewal of ADNI-1 in 2011, termed ADNI-2, enrolled 150 elderly controls, 100 early MCI, 150 late MCI and $150 \mathrm{AD}$ patients in addition to existing ADNI-1 and ADNI-GO cohorts. Successive studies added new technologies such as fMRI, amyloid imaging using ${ }^{11} \mathrm{C}$-Pittsburg compound, and then ${ }^{18} \mathrm{~F}$ AV45 radioligands, and MRI techniques to detect microhemorrhages. Some subjects have now been monitored for 10 years. providing critical longitudinal data.

\section{Data sharing}

A groundbreaking feature of ADNI from its beginning was the resolve to make all data generated available to qualified researchers worldwide. This open data sharing was unprecedented for NIH funded studies To achieve this, the Informatics core constructed a sophisticated infrastructure based at the Laboratory of Neuroimaging, currently at the University of Southern California, to facilitate the storage, curating and sharing of ADNI imaging, biomarker, clinical, and genetic data (6).

\section{Relationships between biomarkers}

ADNI data has been used in more than 600 publications spanning multiple scientific areas ranging from epidemiology to computer science to genetics and beyond. Two early studies established a biomarker 'signature' for AD that defined a series of cutpoints for CSF biomarkers beyond which a patient would be considered to have a high probability of the disease $(7,8)$. Combining low $A \beta_{42}$ and high t-tau or $\mathrm{p}-\mathrm{tau}_{181}$ levels, these signatures identified AD pathology in AD patients as expected (7), but also suggested that cognitively normal participants could harbor abnormal tau and $A \beta$ brain pathology (8). Early longitudinal biomarker data contributed to a provocative model for the temporal ordering of pathophysiological changes occurring during the progression of AD by Jack et al (9), which has been subsequently refined based on analysis of further ADNI data $(10,11)$, and which is 
supported by many studies (12). Perhaps the most influential ADNI paper, the Jack et al temporal ordering of ADNI biomarkers (9) is the most widely accepted working model of $\mathrm{AD}$ biomarker dynamics.

\section{Diagnosis}

The rich and continually growing ADNI data set has provided opportunities for researchers to investigate new approaches to the challenges of diagnostic classification and the prediction of future clinical change in subjects ranging from those who are cognitively normal to those with MCI or who have manifest AD. Vemuri et al first combined MRI and CSF biomarkers for AD diagnosis (13) and the prediction of future clinical decline (14), finding that CSF biomarkers increased the diagnostic accuracy and predictive ability of MRI measures. Subsequently, a multimodal classifier that selected MRI and FDG PET regions of interest and combined them with CSF biomarker data proved highly accurate (15). This marked the transition to the use of the full breadth of ADNI data for these tasks and recent studies have continued to improve accuracy by focusing on the selection of features that are most AD-like across multiple modalities and by incorporating longitudinal data (12).

\section{Genetics}

As of the end of 2014, there were 2065 distinct $A P O E$ genotype data results available, and GWAS data were available for 1252 participants within ADNI. Over 200 publications have used ADNI genetic data, representing a contribution to $\mathrm{AD}$ genetics that extends far beyond the original mandate of the initiative. In 2009, Potkin et al (16) reported the first ADNI genome-wide association study (GWAS) using MRI hippocampal volume as quantitative trait. Since then, the ADNI Genetics core has pioneered the first GWAS using measures of CSF A $\beta$ and tau (17), whole brain ROIs (18), longitudinal hippocampal MRI volumetric change (19), and A $\beta$ amyloid deposition measured by Florbetapir PET (20) as quantitative traits, the first voxel-based GWAS (21), and the first whole-exome sequencing study in MCI patients (22). Significantly, ADNI genetic data have been part of larger data sets that have identified novel variants for $\mathrm{AD}$ and confirmed previously identified variants (23-25).

\section{Cognition}

Cognition research within ADNI was the focus of a special issue of Brain Imaging and Behavior published in 2012 (26). This issue covered a variety of topics such as psychometric analysis of cognitive tests to improve reliability, sensitivity, and validity of measurements of different cognitive domains, neuroimaging-cognitive relationships, genetic contributions to cognition, and the sequencing of biomarker and cognitive changes in disease progression (26).

\section{Previous Special Issues and Reviews of all ADNI Papers}

Progress made over the course of ADNI-1 was highlighted in a special issue of Alzheimer's \& Dementia in 2010 introduced by Weiner et al (5). This included a set of papers which described the achievements of individual ADNI cores as well as a paper outlining the perspectives of the Private Partner Scientific Board. A second special issue highlighting the 
work of ADNI was also published in 2010 by Neurobiology and Aging. Introduced by Frisoni et al (27), it contained 19 papers on topics related to biomarker dynamics and AD pathophysiology, the prediction of future cognitive decline in MCI and cognitively normal patients, as well as strategies to improve clinical trial design - based on ADNI studies. As ADNI data continued to result in a bounty of publications, the need to summarize the progress made by ADNI in a review format for the benefit of researchers in the field became apparent. In response to this need, Weiner et al reviewed all papers published using ADNI data until the end of 2010 (28), mid-2012 (12), and the end of 2013 (29). The overall impact of ADNI in a myriad of areas has recently been addressed (30).

\section{Standardization}

At the outset of ADNI, a major hurdle was the development of standardized methods that would allow the comparison of results from multiple centers and scanner-hardware variations within the initiative. A major collaborative effort produced a set of protocols for PET and MRI procedures, and the analysis of CSF biomarkers (available at http:// adni.loni.usc.edu/methods/) (5). These have proved essential beyond the scope of ADNI. The standardized protocols have been employed in virtually all clinical trials by pharmaceutical companies developing disease modifying treatments for AD and most recently AD prevention trials (30). There are also a number of initiatives modelled on ADNI. Worldwide ADNI (WW-ADNI), sponsored by the Alzheimer's Association, has established eight similar initiatives in Europe, Asia, Australia, and South America which aim to track AD progression in diverse ethnic groups, and from which standardized data are available to the international research community (31). Other initiatives based on ADNI investigate both risk factors for AD and the pathophysiology of other neurodegenerative diseases. Currently, three programs are investigating post-traumatic stress disorder and traumatic brain injury in Vietnam veterans (32), and depression (30) as risk factors for AD. Other programs are aimed at identifying biomarkers for PD (33), frontotemporal lobar degeneration (30), multiple sclerosis (34), and Down's syndrome (30).

\section{Amyloid PET and Tau PET}

One distinguishing feature of ADNI is its incorporation of developing technologies in successive grant cycles. For example, A $\beta$ amyloid PET imaging initially used the Pittsburgh compound ligand in ADNI-1, but included the Florbetapir ligand in ADNI-2, which was labelled with a longer lived isotope $\left({ }^{18} \mathrm{~F}\right.$ versus $\left.{ }^{11} \mathrm{C}\right)$ and therefore increases imaging practicality. Currently, ADNI has been granted funding to conduct tau amyloid PET studies using a recently developed ligand that detects tau tangles in the living brain. Data from this pilot study is expected to contribute to a competitive renewal of ADNI-2, termed ADNI-3, which if funded, would begin in 2016 and would include tau amyloid PET in addition to current protocols in a five-year study.

The first decade of ADNI has been characterized by outstanding innovation and progress. ADNI's major high impact contributions have been the validation of diverse imaging and chemical biomarkers for use in subject selection and as surrogate outcome measures in AD clinical trials, the standardization of protocols for use in multicenter settings, and the 
establishment of a successful large-scale data sharing infrastructure which has in turn contributed to the publication of over 600 scientific papers. Highly significant ADNI publications have described a widely accepted model for biomarker dynamics in AD pathogenesis, established a biomarker 'signature' for AD, leveraged ADNI's rich multimodal dataset for diagnostic classification and prediction of future decline, and include numerous GWAS and other genetic studies identifying or confirming novel AD risk alleles. The success of ADNI in pioneering funding approaches, data-sharing and the standardization of methods is evidenced by similar initiatives that are now investigating distinct risk factors for AD as well as developing biomarkers for other diseases such as Parkinson's disease. It is expected that the continuation of the study in the next decade will result in a further proliferation of technological advances and improvements to clinical trial efficiency, and in an even deeper understanding of AD pathophysiology.

\section{Acknowledgments}

This work was supported by NIH grant 5U01AG024904-10 funded by the National Institute on Aging to Dr Michael Weiner.

Michael W. Weiner has served on the scientific advisory boards for Lilly, Araclon and Institut Catala de Neurociencies Aplicades, Gulf War Veterans Illnesses Advisory Committee, VACO, Biogen Idec, and Pfizer; has served as a consultant for Astra Zeneca, Araclon, Medivation/Pfizer, Ipsen, TauRx Therapeutics LTD, Bayer Healthcare, Biogen Idec, Exonhit Therapeutics, SA, Servier, Synarc, Pfizer, and Janssen; has received funding for travel from NeuroVigil, Inc., CHRU-Hopital Roger Salengro, Siemens, AstraZeneca, Geneva University Hospitals, Lilly, University of California, San Diego - ADNI, Paris University, Institut Catala de Neurociencies Aplicades, University of New Mexico School of Medicine, Ipsen, CTAD (Clinical Trials on Alzheimer's Disease), Pfizer, AD PD meeting, Paul Sabatier University, Novartis, Tohoku University; has served on the editorial advisory boards for Alzheimer's \& Dementia and MRI; has received honoraria from NeuroVigil, Inc., Insitut Catala de Neurociencies Aplicades, PMDA/Japanese Ministry of Health, Labour, and Welfare, and Tohoku University; has received commercial research support from Merck and Avid; has received government research support from DOD and VA; has stock options in Synarc and Elan; and declares the following organizations as contributors to the Foundation for $\mathrm{NIH}$ and thus to the NIA funded Alzheimer's Disease Neuroimaging Initiative: Abbott, Alzheimer's Association, Alzheimer's Drug Discovery Foundation, Anonymous Foundation, AstraZeneca, Bayer Healthcare, BioClinica, Inc. (ADNI 2), Bristol-Myers Squibb, Cure Alzheimer's Fund, Eisai, Elan, Gene Network Sciences, Genentech, GE Healthcare, GlaxoSmithKline, Innogenetics, Johnson \& Johnson, Eli Lilly \& Company, Medpace, Merck, Novartis, Pfizer Inc., Roche, Schering Plough, Synarc, and Wyeth.

\section{References}

1. Khachaturian ZS. Toward a comprehensive theory of Alzheimer's disease-challenges, caveats, and parameters. Annals of the New York Academy of Sciences. 2000; 924:184-93. [PubMed: 11193796]

2. Petersen RC. Mild cognitive impairment: transition between aging and Alzheimer's disease. Neurologia. 2000; 15(3):93-101. [PubMed: 10846869]

3. Mueller SG, Weiner MW, Thal LJ, Petersen RC, Jack C, Jagust W, et al. The Alzheimer's disease neuroimaging initiative. Neuroimaging Clin N Am. 2005; 15(4):869-77. xi-xii. [PubMed: 16443497]

4. Mueller SG, Weiner MW, Thal LJ, Petersen RC, Jack C, Jagust W, et al. Ways toward an early diagnosis in Alzheimer's disease: the Alzheimer's Disease Neuroimaging Initiative. Cognition and Dementia. 2006; 5(4):56-62.

5. Weiner MW, Aisen PS, Jack CR Jr, Jagust WJ, Trojanowski JQ, Shaw L, et al. The Alzheimer's disease neuroimaging initiative: progress report and future plans. Alzheimers Dement. 2010; 6(3): 202-11. e7. [PubMed: 20451868]

6. Toga AW, C K. The informatics core of the Alzheimer's Disease Neuroimaging Initiative. Alzheimers Dement. 2010; 6(3):247-56. [PubMed: 20451873] 
7. Shaw LM, Vanderstichele H, Knapik-Czajka M, Clark CM, Aisen PS, Petersen RC, et al. Cerebrospinal fluid biomarker signature in Alzheimer's disease neuroimaging initiative subjects. Ann Neurol. 2009; 65(4):403-13. [PubMed: 19296504]

8. De Meyer G, Shapiro F, Vanderstichele H, Vanmechelen E, Engelborghs S, De Deyn PP, et al. Diagnosis-Independent Alzheimer Disease Biomarker Signature in Cognitively Normal Elderly People. Arch Neurol. 2010; 67(8):949-56. [PubMed: 20697045]

9. Jack CR Jr, Knopman DS, Jagust WJ, Shaw LM, Aisen PS, Weiner MW, et al. Hypothetical model of dynamic biomarkers of the Alzheimer's pathological cascade. Lancet Neurol. 2010; 9(1):119-28. [PubMed: 20083042]

10. Jack CR Jr, Vemuri P, Wiste HJ, Weigand SD, Aisen PS, Trojanowski JQ, et al. Evidence for ordering of Alzheimer disease biomarkers. Arch Neurol. 2011; 68(12):1526-35. [PubMed: 21825215]

11. Jack CR Jr, Knopman DS, Jagust WJ, Petersen RC, Weiner MW, Aisen PS, et al. Tracking pathophysiological processes in Alzheimer's disease: an updated hypothetical model of dynamic biomarkers. Lancet Neurol. 2013; 12(2):207-16. [PubMed: 23332364]

12. Weiner MW, Veitch DP, Aisen PS, Beckett LA, Cairns NJ, Green RC, et al. The Alzheimer's Disease Neuroimaging Initiative: a review of papers published since its inception. Alzheimers Dement. 2013; 9(5):e111-94. [PubMed: 23932184]

13. Vemuri P, Wiste HJ, Weigand SD, Shaw LM, Trojanowski JQ, Weiner MW, et al. MRI and CSF biomarkers in normal, MCI, and AD subjects: diagnostic discrimination and cognitive correlations. Neurology. 2009; 73(4):287-93. [PubMed: 19636048]

14. Vemuri P, Wiste HJ, Weigand SD, Shaw LM, Trojanowski JQ, Weiner MW, et al. MRI and CSF biomarkers in normal, MCI, and AD subjects: predicting future clinical change. Neurology. 2009; 73(4):294-301. [PubMed: 19636049]

15. Zhang D, Wang Y, Zhou L, Yuan H, Shen D. Multimodal classification of Alzheimer's disease and mild cognitive impairment. Neuroimage. 2011; 55(3):856-67. [PubMed: 21236349]

16. Potkin SG, Guffanti G, Lakatos A, Turner JA, Kruggel F, Fallon JH, et al. Hippocampal atrophy as a quantitative trait in a genome-wide association study identifying novel susceptibility genes for Alzheimer's disease. PLoS One. 2009; 4(8):e6501. [PubMed: 19668339]

17. Han MR, Schellenberg GD, Wang LS. Genome-wide association reveals genetic effects on human Abeta42 and tau protein levels in cerebrospinal fluids: a case control study. BMC Neurol. 2010; 10:90. [PubMed: 20932310]

18. Shen L, Kim S, Risacher SL, Nho K, Swaminathan S, West JD, et al. Whole genome association study of brain-wide imaging phenotypes for identifying quantitative trait loci in MCI and AD: A study of the ADNI cohort. Neuroimage. 2010; 53(3):1051-63. [PubMed: 20100581]

19. Furney SJ, Simmons A, Breen G, Pedroso I, Lunnon K, Proitsi P, et al. Genome-wide association with MRI atrophy measures as a quantitative trait locus for Alzheimer's disease. Mol Psychiatry. 2011; 16(11):1130-8. [PubMed: 21116278]

20. Ramanan VK, Risacher SL, Nho K, Kim S, Swaminathan S, Shen L, et al. APOE and BCHE as modulators of cerebral amyloid deposition: a florbetapir PET genome-wide association study. Mol Psychiatry. 2013

21. Stein JL, Hua X, Lee S, Ho AJ, Leow AD, Toga AW, et al. Voxelwise genome-wide association study (vGWAS). Neuroimage. 2010; 53(3):1160-74. [PubMed: 20171287]

22. Nho K, Corneveaux JJ, Kim S, Lin H, Risacher SL, Shen L, et al. Whole-exome sequencing and imaging genetics identify functional variants for rate of change in hippocampal volume in mild cognitive impairment. Mol Psychiatry. 2013; 18(7):781-7. [PubMed: 23608917]

23. Naj AC, Jun G, Beecham GW, Wang LS, Vardarajan BN, Buros J, et al. Common variants at MS4A4/MS4A6E, CD2AP, CD33 and EPHA1 are associated with late-onset Alzheimer's disease. Nat Genet. 2011; 43(5):436-41. [PubMed: 21460841]

24. Lambert JC, Ibrahim-Verbaas CA, Harold D, Naj AC, Sims R, Bellenguez C, et al. Meta-analysis of 74,046 individuals identifies 11 new susceptibility loci for Alzheimer's disease. Nat Genet. 2013; 45(12):1452-8. [PubMed: 24162737] 
25. Hollingworth P, Harold D, Sims R, Gerrish A, Lambert JC, Carrasquillo MM, et al. Common variants at ABCA7, MS4A6A/MS4A4E, EPHA1, CD33 and CD2AP are associated with Alzheimer's disease. Nat Genet. 2011; 43(5):429-35. [PubMed: 21460840]

26. Mungas D, Crane PK, Gibbons LE, Manly JJ, Glymour MM, Jones RN. Advanced psychometric analysis and the Alzheimer's Disease Neuroimaging Initiative: reports from the 2011 Friday Harbor conference. Brain Imaging Behav. 2012; 6(4):485-8. [PubMed: 23232798]

27. Frisoni GB, Weiner MW. Alzheimer's Disease Neuroimaging Initiative special issue. Neurobiol Aging. 2010; 31(8):1259-62. [PubMed: 20570400]

28. Weiner MW, Veitch DP, Aisen PS, Beckett LA, Cairns NJ, Green RC, et al. The Alzheimer's Disease Neuroimaging Initiative: a review of papers published since its inception. Alzheimers Dement. 2012; 8(1 Suppl):S1-68. [PubMed: 22047634]

29. Weiner MW, Veitch DP, Aisen PS, Beckett LA, Cairns NJ, Cedarbaum J, Green RC, Harvey D, Jack CR, Jagust W, Luthman J, Morris JC, Petersen RC, Saykin AJ, Shaw L, Shen L, Schwarz A, Toga AW, Trojanowski JQ. Alzheimer's Disease Neuroimaging Initiative. 2014 update of The Alzheimer's Disease Neuroimaging Initiative: A review of papers published since its inception. Alzheimer's \& Dementia. 2015

30. Weiner MW, Veitch DP, Aisen PS, Beckett LA, Cairns NJ, Cedarbaum J, Donohue MC, Green RC, Harvey D, Jack CR, Jagust W, Morris JC, Petersen RC, Saykin AJ, Shaw L, Shen L, Schwarz A, Thmpson PM, Toga AW, Trojanowski JQ. Impact of the Alzheimer's Disease Neuroimaging Initiative, 2004-2014. Alzheimer's \& Dementia. 2015

31. Carrillo MC, Bain LJ, Frisoni GB, Weiner MW. Worldwide Alzheimer's disease neuroimaging initiative. Alzheimers Dement. 2012; 8(4):337-42. [PubMed: 22748939]

32. Weiner MW, Veitch DP, Hayes J, Neylan T, Grafman J, Aisen PS, et al. Effects of traumatic brain injury and posttraumatic stress disorder on Alzheimer's disease in veterans, using the Alzheimer's Disease Neuroimaging Initiative. Alzheimers Dement. 2014; 10(3 Suppl):S226-35. [PubMed: 24924673]

33. Parkinson Progression Marker I. The Parkinson Progression Marker Initiative (PPMI). Progress in neurobiology. 2011; 95(4):629-35. [PubMed: 21930184]

34. Rammohan KW. Transformation of MS care in the 21st Century. How NARCRMS will change the way we practice. 6th Cooperative Meeting of the Consortium of Multiple Sclerosis Centers (CMSC) and the Americas Committee for Treatment and Research In Multiple Sclerosis (ACTRIMS). 2014 